\title{
Orientações sobre parto no pré-natal de alto risco nos serviços de saúde
}

Guidelines on high-risk prenatal delivery in health services

Orientación sobre el parto prenatal de alto riesgo en los servicios de salud

\section{Juliana Carvalho Lourenço ${ }^{\mathrm{I}}$, Fabiana Fontana Medeiros ${ }^{\mathrm{II}}$, Mariana Haddad Rodrigues ${ }^{\mathrm{III}}$ Rosângela Aparecida Pimenta Ferrari ${ }^{\mathrm{IV}}$, Deise Serafimv, Alexandrina Aparecida Maciel Cardelli ${ }^{\mathrm{VI}}$}

Resumo: Objetivo: desvelar as orientações recebidas sobre parto durante o pré-natal de alto risco nos serviços de saúde à luz da teoria das representações sociais. Método: estudo descritivo exploratório com abordagem qualitativa, tendo a Teoria das Representações Sociais como referencial teórico metodológico. A coleta de dados ocorreu entre outubro de 2017 e janeiro de 2018, em uma maternidade do norte do Paraná, durante a internação de 20 puérperas. Utilizou-se entrevista semiestruturada e análise de conteúdo temática. Resultados: emergiu-se a categoria: conhecimento elaborado frente as orientações em saúde para gestantes. O núcleo central configurou-se no cuidado direcionado à saúde da mulher e seu empoderamento no ciclo gravídico-puerperal. Conclusão: orientações em saúde foram similares nos dois níveis assistenciais, sendo direcionadas à saúde e bem-estar fetal. Foram identificadas situações de risco ao bebê nas urgências que poderiam resultar em um parto cesáreo prematuro e complicações ao concepto.

Descritores: Gravidez de alto risco; Serviços de saúde; Cuidado pré-natal; Educação em saúde, Saúde da mulher

Abstract: Objective: to unveil the guidance received on childbirth during high-risk prenatal care in health services in the light of Social Representations Theory. Method: an exploratory descriptive study with qualitative approach, with the Social Representations Theory as a methodological theoretical framework. Data collection occurred between October 2017 and January 2018, in a maternity hospital in northern Paraná, during the hospitalization of 20 puerperal women. Semi-structured interviews and thematic content analysis were used. Results: the category emerged: Knowledge developed in the face of health guidelines for pregnant women. The

\footnotetext{
${ }^{I}$ Enfermeira. Mestra em Enfermagem pela Universidade Estadual de Londrina/UEL. Londrina (PR) - Brasil. E-mail: julilou26@hotmail.com ORCID ID: https://orcid.org/0000-0001-7854-8074

II Enfermeira. Doutoranda do Programa de Pós-Graduação em Enfermagem da Universidade Estadual de Londrina/UEL. Londrina (PR) - Brasil. E-mail: fontana.fabi@hotmail.com - ORCID ID: https://orcid.org/0000-0002-7876-572

${ }^{\text {III }}$ Enfermeira. Pós-Doutoranda do Programa de Pós-Graduação em Enfermagem da Universidade Estadual de Londrina/UEL. Londrina (PR) Brasil. E-mail: mari.tradu@gmail.com - ORCID ID: https://orcid.org/0000-0002-8339-9760

${ }^{\text {IV }}$ Enfermeira. Doutora em Enfermagem. Professora Associada do Departamento de Enfermagem da Universidade Estadual de Londrina/UEL. Londrina (PR) - Brasil. E-mail: ropimentaferrari@uel.br - ORCID ID: https://orcid.org/0000-0003-0157-7461

${ }^{\vee}$ Enfermeira. Doutora em Saúde Pública. Professora Adjunta do Departamento de Enfermagem da Universidade Estadual de Maringá/UEM. Maringá (PR) - Brasil. E-mail: dserafim@hotmail.com - ORCID ID: https://orcid.org/0000-0001-5681-8387

VI Enfermeira. Doutora em Saúde Pública. Professora Associada do Departamento de Enfermagem da Universidade Estadual de Londrina/UEL. Londrina (PR) - Brasil. E-mail: macielalexandrina@gmail.com - ORCID ID https://orcid.org/0000-0002-0222-8821
} 
central nucleus was configured in care directed to women's health and their empowerment in the pregnancypuerperal cycle. Conclusion: health guidelines were similar at both levels of care, being directed to fetal health and well-being. Risk situations to newborns were identified in the emergency room that could result in premature cesarean delivery and complications to the conceptus.

Descriptors: Pregnancy, high-risk; Health services; Prenatal care; Health education; Women’s health

Resumen: Objetivo: dar a conocer las pautas recibidas sobre el parto durante la atención prenatal de alto riesgo en los servicios de salud a la luz de la teoría de las representaciones sociales. Método: estudio exploratorio descriptivo con abordaje cualitativo, con la Teoría de las Representaciones Sociales como marco teórico y metodológico. La recolección de datos se llevó a cabo entre octubre de 2017 y enero de 2018, en una maternidad del norte de Paraná, durante la hospitalización de 20 madres. Se utilizaron entrevistas semiestructuradas y análisis de contenido temático. Resultados: surgió la categoría: Conocimientos elaborados frente a guías de salud para gestantes. El núcleo central se configuró en la atención dirigida a la salud de la mujer y su empoderamiento en el ciclo embarazo-puerperal. Conclusión: las pautas de salud fueron similares en los dos niveles de atención, dirigidas a la salud y el bienestar fetal. Se identificaron situaciones de riesgo para el bebé en emergencias que podrían resultar en un parto por cesárea prematuro y complicaciones para el feto.

Descriptores: Embarazo de alto riesgo; Servicios de salud; Atención prenatal; Educación en salud; Salud de la mujer

\section{Introdução}

A gestação é um fenômeno fisiológico que envolve mudanças dinâmicas, físico, social e emocional. No entanto, o período gestacional pode resultar em riscos tanto para a mulher quanto ao feto, assim como a própria característica da mulher pode apresentar em maior probabilidade para desfechos desfavoráveis, sendo consideradas gestantes de alto risco. ${ }^{1}$

A atenção pré-natal destaca-se como fator essencial para saúde das mulheres no ciclo gravídico-puerperal. Práticas realizadas de maneira adequada durante esse acompanhamento resultam em melhores desfechos maternos e perinatais. A realização de forma inadequada dessa assistência tem sido relacionada com altos índices de morbimortalidade materna e infantil. ${ }^{2}$

De acordo com as recomendações do Ministério da Saúde, a assistência pré-natal deve partir de condutas acolhedoras, com desenvolvimento de ações educativas e preventivas, sem intervenções desnecessárias, com detecção precoce de patologias e de situações de risco gestacional. A gestante tem o direito de estabelecer vínculo entre o local de realização do pré- 
natal e a instituição que realizará o seu parto, desde o atendimento ambulatorial básico ao atendimento hospitalar de alto risco, além da garantia de fácil acesso a serviços de qualidade. ${ }^{3}$

A Política Nacional de Humanização $(\mathrm{PNH})$ tem proposto metodologias de atenção e gestão dos programas e estratégias existentes na saúde, prevendo a inclusão de todos os atores sociais envolvidos neste processo. No âmbito do nascimento, o programa de saúde Rede Cegonha propõe uma rede de cuidados que garanta assistência humanizada e de qualidade à mulher. ${ }^{4}$

Nos últimos anos, o Brasil teve um avanço importante relacionado a programas para saúde da mulher no ciclo gravídico puerperal. No entanto, há lacunas à assistência gestacional a serem preenchidas, como a persistência da falta de visão integral sobre a saúde da mulher, que resulta na fragmentação das informações ou cuidados durante o pré-natal. ${ }^{5}$

No Brasil, entre os anos de 2009 e 2013, ocorreram 8.470 óbitos maternos, apresentando uma razão de mortalidade materna de 58.55 óbitos a cada 100 mil nascidos vivos, por alguma causa relacionada à gestação e ao parto. Entretanto, 95\% desses óbitos poderiam ser evitados se tivessem recebido assistência adequada. ${ }^{6}$

A educação em saúde, com orientações sobre cuidados e higiene, alimentação, modificações corporais, atividade sexual, atividade física, parto, cuidados com o recém-nascido e aleitamento materno, é importante para a gestante. Porém, em estudo recente, constata-se que a maioria das mulheres não recebe esse tipo de informação. ${ }^{7}$

Uma pesquisa de base hospitalar com 23.894 mulheres, entre 2011 e 2012, desvelou que as diferenças, segundo o tipo de serviço de saúde, público ou privado, não foram observadas após ajuste das características maternas. A inexistência de diferença revela que, ao eleger um critério de adequação global mínima, serviços públicos e privados demonstrariam o mesmo grau de adequação se atendessem pacientes com características sociais e econômicas similares. ${ }^{8}$ 
O conhecimento do senso comum sobre o acompanhamento pré-natal nos serviços privados sugere que esse cuidado, muitas vezes não segue as recomendações do Ministério da Saúde quanto ao número de consultas e ao que deve ser orientado durante o pré-natal. Vale ressaltar que a equipe multidisciplinar nesse tipo de serviço existe, porém, somente quando há indicação ocorre o encaminhamento pelo médico obstetra ou procura direta pela paciente.

Observa-se uma lacuna na prática profissional sobre as orientações essenciais, para que gestantes de alto risco estejam preparadas para enfrentar o momento do parto. Nesse sentido, há necessidade de compreender as orientações recebidas sobre esse momento no pré-natal de alto risco, nos diferentes serviços de saúde, para contribuição ao período do nascimento, o qual pode resultar em desfechos favoráveis ou não. O presente estudo teve por objetivo desvelar as orientações recebidas sobre parto durante o pré-natal de alto risco nos serviços de saúde à luz da teoria das representações sociais.

Utilizamos a Teoria das Representações Sociais por sua característica em relação à busca do conhecimento elaborado no fenômeno “orientações para o parto”, onde o grupo social de mulheres de alto risco pode almejar compreender o momento do nascimento durante o prénatal.

\section{Método}

Trata-se de um estudo descritivo exploratório com abordagem qualitativa, tendo a Teoria das Representações Sociais como referencial teórico metodológico. ${ }^{9}$ A representação social tem como objetivo integrar um fenômeno social, o qual não faz parte do cotidiano e que pode refletir no medo e ansiedade dos indivíduos e grupos. Entretanto, à medida que o referencial conceitual é incorporado, torna-se familiar e é reelaborado, resultando em uma nova forma de conhecimento de um grupo social, chamado saber do senso comum. ${ }^{10}$ 
5 | Lourenço JC, Medeiros FF, Rodrigues MH, Ferrari RAP, Serafim D, Cardelli AAM

Define-se a representação social como um conjunto de conceitos, frases e explicações originadas na vida diária durante o curso das comunicações interpessoais. Dessa maneira, as representações são fenômenos sociais entendidos a partir do seu contexto de produção, isto é, desde as funções simbólicas e ideológicas a que servem e das formas de comunicação onde circulam. $^{9}$

Desde a gestação até o momento do parto, a mulher pode enfrentar dúvidas e anseios. Nesse sentido, a representação social construída ao longo da gestação, pode refletir para a reelaboração desses conceitos até o momento do parto, trazendo a elaboração do conhecimento da própria mulher.

O estudo foi realizado em uma maternidade de um hospital filantrópico do norte do Paraná, de outubro de 2017 a janeiro de 2018. A maternidade contava com sistema de alojamento conjunto com 46 leitos, sendo 13 (28\%) destinados somente para gestação de alto risco, vinculados ao Sistema Único de Saúde (SUS), e os demais para atendimentos (com convênio ou particular) clínicos em geral e partos utilizados tanto para o risco habitual como para o alto risco.

Os critérios de inclusão do estudo foram: ter idade mínima de 18 anos, ter como produto de concepção recém-nascido vivo e ter um dos critérios para gestação de alto risco, conforme a Linha Guia Rede Mãe Paranaense. ${ }^{11}$ Consideraram-se os seguintes critérios de exclusão: mãe sedada ou em ventilação mecânica.

Para a seleção das participantes, buscou-se paridade da amostra quanto às participantes do serviço público e serviço privado, alcançando os dois públicos. Nesse sentido, a escolha das participantes ocorreu previamente de acordo com os critérios de inclusão do estudo e a paridade para os dois serviços.

Os dados foram coletados pela própria pesquisadora, a qual possui mestrado em obstetrícia, ocupando cargo de supervisão na maternidade em estudo. Realizou-se teste piloto 
Orientações sobre parto no pré-natal de alto risco nos serviços de saúde | 6

com três puérperas, sendo adequado o formulário semiestruturado, identificando-se características socioeconômicas e demográficas, situação conjugal, escolaridade, condição de ocupação, idade e ao convênio de saúde. Após, realizou-se entrevista com a pergunta norteadora: durante o pré-natal você recebeu orientações sobre o momento do seu parto? Utilizou-se o critério de saturação dos dados. ${ }^{12}$ Quando necessário, a pesquisadora utilizava as seguintes subquestões para obter maiores esclarecimentos: como foi a orientação? Você poderia falar mais sobre a orientação? Falou-se sobre o parto durante o pré-natal?

Cada entrevista teve, em média, duração de sessenta minutos, o qual foi oportunizado devido a pesquisadora ocupar o cargo de supervisora na maternidade em estudo, onde organizou-se a equipe de enfermagem ao apoio no cuidado ao recém-nascido. As entrevistas ocorreram durante a internação em momento oportuno definido pelas puérperas, após vinte e quatro horas pós-parto, em local reservado estando presente somente a puérpera e entrevistadora.

Utilizou-se gravador de voz, e as entrevistas foram transcritas diariamente após a coleta de dados. Houve correção na transcrição do português e vícios de linguagem, não comprometendo o sentido das falas. Após, procedeu-se a interpretação dos dados pela análise de conteúdo temática, delineada pelas seguintes fases: pré-análise, exploração do material e tratamento dos resultados. ${ }^{13}$

Os depoimentos das puérperas foram identificados de um a vinte, sendo codificado conforme o serviço nos quais foram atendidas: PSUS (para as gestantes que realizaram o prénatal no serviço público) e PRI (para as gestantes que realizaram o pré-natal no serviço particular/convênio), a fim de manter o sigilo das participantes. Não houve recusa entre as entrevistadas do estudo.

Este estudo seguiu as recomendações éticas da Resolução 466/2012, da Comissão Nacional de Saúde e foi aprovado pelo Comitê de Ética em Pesquisa Evolvendo Seres Humanos 
da Universidade Estadual de Londrina (CEP/UEL), em 09 de outubro de 2017, sob Parecer: 2.323.783 e CAAE: 78643417400005231. As participantes foram informadas previamente, por meio do Termo de Consentimento Livre e Esclarecido, sobre os objetivos e benefícios da pesquisa para os serviços de saúde, dando início às entrevistas somente após aceite e assinatura do termo.

\section{Resultados}

Tendo como pressuposto a Teoria das Representações Sociais, a análise sobre as orientações recebidas, durante o pré-natal, tanto no serviço de saúde público como no privado, revelou que as mulheres receberam informações direcionadas à saúde e bem-estar fetal, bem como sobre a identificação de situações de risco para o bebê, evidenciadas como urgências que poderiam levar a um parto cesáreo prematuro e complicações para o concepto. Por outro lado, alguns depoimentos desvelaram que as orientações recebidas não supriram as necessidades das gestantes, levando-as a procurar informações em outras fontes na internet.

Participaram do estudo vinte puérperas, dessas doze usuárias da saúde pública e oito do serviço privado. Três participantes frequentaram o pré-natal nos dois convênios, sendo que duas finalizaram o pré-natal no serviço privado, e uma mulher realizou todo pré-natal no convênio, mas o parto foi realizado no público. Essas três puérperas foram consideradas como usuárias do serviço privado. Entre as entrevistadas, sete mulheres realizaram pré-natal no serviço especializado para gestante de alto risco, e continuaram o acompanhamento na Atenção Primária à Saúde. As demais foram atendidas somente pela Unidade Básica de Saúde, mas foram classificadas como gestantes de alto risco pela unidade que realizou o acompanhamento.

Os critérios para o acompanhamento pré-natal de alto risco das participantes do estudo foram: hipertensão arterial crônica, diabetes mellitus gestacional, hipotireoidismo, trabalho de parto prematuro, amniorrexe prematuro, retardo do crescimento intraútero, oligodramnia, 
cirurgia bariátrica e obesidade. Quanto à via de parto das participantes, sete foram parto vaginal, dessas seis do serviço público e um do privado e treze cesarianas, sendo seis da saúde púbica e sete do serviço privado. A caracterização socioeconômica demográfica ocorreu com mulheres desde os 18 até os 40 anos de idade. Treze com ensino médio, dez tinham algum tipo de ocupação e dezessete possuíam companheiro.

A partir da questão norteadora Durante o pré-natal você recebeu orientações sobre o momento do seu parto?, pode-se desvelar que o núcleo central configurou no cuidado direcionado à saúde da mulher e seu empoderamento no ciclo gravídico-puerperal. Conhecimento elaborado frente as orientações em saúde para gestantes resultaram na categoria temática após análise dos depoimentos.

\section{Conhecimento elaborado frente as orientações em saúde para gestantes}

Nesta categoria, quando as mulheres foram questionadas acerca das orientações recebidas durante o pré-natal, sobre o momento do parto, as respostas revelaram diferentes aspectos abordados pelos profissionais, entre eles: riscos para o bebê, via de parto e informações sobre internação. Em contrapartida, alguns depoimentos demonstraram a falta de informações que suprissem a curiosidade das gestantes sobre como seria de fato o seu parto. Perceberam-se, ainda, diferenças determinadas pelo sistema no qual a gestante foi atendida, sendo serviço de saúde público e privado.

Durante o seguimento pré-natal de alto risco no serviço público, houve orientações sobre os riscos do último mês, e de como proceder, na ocorrência de sinais ou sintomas descritos na consulta pré-natal, tais como o aumento da pressão arterial e possibilidade de laqueadura, em caso de parto cirúrgico. 
É, no ambulatório de alto risco ela disse que ia ser cesárea, pela pressão, e que eu tinha que fazer laqueadura [...]. Se a minha pressão continuasse alterando do jeito que alterou o tempo todo, depois, no último mês, poderia dar uma [...] pré-eclâmpsia. (PSUS2)

Algumas mulheres do serviço público foram informadas sobre vias de parto, recebendo orientações sobre o tempo de trabalho de parto, o ritmo das contrações e dor. A prematuridade e a diabetes gestacional também foram temas abordados durante as consultas como possíveis complicações que impedissem a via de parto vaginal.

A partir do meio, mais ou menos, o pré-natal, da quarta consulta a gente já começou a conversar sobre parto e ele falou que é doloroso, falou do ritmado das contrações [...] mais ou menos o tempo que duraria, dependia da evolução que eu teria. Falou que, dependendo, se eu tivesse diabetes gestacional, meu bebê poderia nascer prematuro. (PSUS17)

As orientações recebidas pelas mulheres que frequentaram o serviço privado foram similares àquelas recebidas no serviço público quanto à justificativa para risco de prematuridade, momento da internação e vias de parto. Notou-se a preocupação do profissional do convênio em orientar a mulher sobre os trâmites burocráticos da internação.

Recebi orientações [...] ele me orientou, me deu todas as papeladas [...] caso a bolsa rompesse, que não era para eu sair correndo, que eu podia chegar a qualquer momento, que era para chegar aqui no hospital e avisar o médico [...] que eu tenho convênio, certinho. Ele falou que o parto seria a tal hora, e que o internamento seria um pouco antes [...]. E como ele sempre teve um percentual alto, sempre foi uma criança acima [...] do esperado, o tamanho, ele falou: "Olha vai judiar muito”. (PRI8)

Percebeu-se também que a mulher atendida no serviço privado teve a oportunidade de realizar visita à maternidade antes do parto e escolher o médico pediatra que recepcionaria o 
recém-nascido. Houve a preocupação com a amamentação no primeiro momento de vida, favorecendo medidas preventivas de um possível parto prematuro.

Sim, recebi. As orientações foram as seguintes, por exemplo [...], que a minha opção pelo parto seria o parto normal, mas o doutor deixou claro que talvez teria intercorrências que não seria possível; que a gente não poderia focar apenas no parto normal, para que quando chegasse o momento, eu não ficasse com aquela expectativa, aquela frustração, aquele medo, tanto é que quando aconteceu a pré-eclâmpsia e eu vim para o hospital e tive que fazer cesariana, foi tudo tão rápido, e, ainda assim, ele me manteve calma, me manteve segura, e ele me explicou tudo. Eu conheci até a maternidade antes do parto. (PRI10)

A gente conversou porque eu tinha preocupação com relação ao atendimento do pediatra, questão de escolha do profissional que ia acompanhar, a questão da amamentação no primeiro momento de vida do bebê[...]. O risco da prematuridade, ele sempre deixou claro, tanto é que ao longo do pré-natal, ele adotou algumas medidas já preventivas com relação à possiblidade de prematuridade. (PRI20)

A apresentação pélvica, o sofrimento fetal, o histórico obstétrico de uma ou mais cirurgias cesarianas e o intervalo interpartal menor que um ano, foram os motivos mencionados pelas mulheres para justificar a indicação de parto operatório. Por meio dos depoimentos, ficou evidente que as mulheres aceitaram a indicação de via de parto sem questionamentos ou dúvidas.

Recebi orientações [...] ele falou que, como eu já tinha tido um parto cesárea, que era melhor eu ter novamente um parto cesárea, e teve um agravante essa segunda gravidez, ele estava sentadinho, então [...]. (PRI8)

Ela já falou, no começo, que seria cesárea, porque eu tenho já duas cesáreas. E a outra tem menos de 1 ano. No último, ela só falou assim, "a 
11 | Lourenço JC, Medeiros FF, Rodrigues MH, Ferrari RAP, Serafim D, Cardelli AAM

gente já vai agendar a sua cesárea”[...]. E que, se tentasse, seria perigoso para mim e para o bebê. (PSUS14)

Desvelaram-se nos depoimentos das mulheres o não recebimento de orientações suficientes e oportunas sobre o momento do parto, como desconhecer a via de parto até a véspera do mesmo.

Não, assim, a gente não sabia como que ia ser [...]. Se poderia ser normal, ou [...] conforme foi caminhando [...] ontem mesmo que foi decidido [...]. Que ia ser cesárea. (PRI1)

Eu nunca cheguei a falar do parto em si, na verdade, a última, penúltima consulta que eu tive com o meu médico, a gente ia conversar sobre o parto [...] só que acabou que aconteceu antes, daí acabou sendo uma cesárea, mas eu sempre pesquisei, sempre busquei, sempre dei umas olhadas, assim [...]. Até falei para doutora, "quero uma explicação sobre o parto”, ela falou [...] "a gente conversa na próxima", e acabou que [...][risos]. (PRI5)

Não chegaram a falar nada, não. Acho que porque eu já tive outras, então [...]. Só perguntavam se a gente estava bem, se não estava tendo sangramento, essas perguntas de rotina que o médico falava. (PSUS18)

No pré-natal, não foi falado não, porque na última consulta que eu tive com ele, eu perguntei se ia ser cesárea ou normal, ele falou que ia esperar o normal porque eu já tive um [...]. A gente tem curiosidade e vai pesquisar na internet, aí eu vi que poderia dar hemorragia, poderia dar as coisas e ele poderia ter falado [...]. Então, muitas coisas eu procurava na internet mesmo. (PSUS19)

A falta de comunicação entre os profissionais que assistem o pré-natal por agravos maternos foi marcante nos depoimentos acima, onde as pacientes se tornam porta-vozes da sua doença. A não informação ocasionou a busca de conhecimento por meios próprios através da internet, a qual foi utilizada para sanar dúvidas e curiosidades. 
Orientações sobre parto no pré-natal de alto risco nos serviços de saúde | 12

\section{Discussão}

Diante de tais interpretações, pode-se apreender o potencial que a representação social tem de criar e transformar a realidade, pois tem a função de expressar a forma como os indivíduos percebem e compreendem um determinado objeto. Assim, pode-se considerar que a representação social é um guia para a ação, por orientar as ações e as relações entre os sujeitos participantes da mesma realidade.

O presente estudo avaliou as orientações sobre o momento do parto no pré-natal de alto risco em uma maternidade de um hospital filantrópico do norte do Paraná, desvelando lacunas sobre este tema, neste cenário. Os achados apontam para uma assistência pré-natal similar do serviço público e privado, evidenciando fragilidades e potencialidades que abrangem os dois convênios.

Em um estudo nacional sobre adequação da assistência pré-natal realizado, com pacientes do serviço público e privado, não foram observadas diferenças entre eles após ajustes das características maternas, alcançando, desta forma, a similaridade dos serviços para adequação da assistência pré-natal. ${ }^{8}$

Em pesquisa realizada em todo o território nacional, incluindo gestantes de risco habitual e alto risco (25\%), identificou-se a deficiência de informações durante a consulta pré-natal quanto à preparação das mulheres para o parto, que priorizou orientações sobre sinais de risco, intensificando, assim, a característica biomédica da assistência materna. ${ }^{14}$

Para as participantes deste estudo, todas classificadas como gestantes de alto risco, as informações sobre sinais de alerta são importantes e devem ser mencionadas durante todo o pré-natal, fato esse justificado por essas mulheres terem maior possibilidade de complicações. Ressalta-se que, quando efetivamente orientadas, elas podem identificar os sinais de risco com prontidão e, assim, buscar os serviços de referência com maior agilidade. 
No presente estudo, desvelaram-se orientações para o momento do parto, com possibilidade de via de parto vaginal ou cesariana, porém não foram disponibilizadas informações sobre as vias de parto com indicações, contraindicações e riscos inerentes a cada uma delas. Percebe-se que a indicação é influenciada pelo profissional para a escolha da cirurgia cesariana, principalmente justificada pelos riscos inerentes à gravidez de alto risco.

Uma pesquisa realizada em um município do Paraná, com resultados semelhantes ao presente estudo, observou uma realidade de que a cada quatro nascimentos, três foram por parto cesariana; quanto às frequências, $65,3 \%$ das cesarianas foram realizadas no SUS e 97,8\% na saúde suplementar. A cesariana deixou de ser um procedimento cirúrgico com objetivo nos resultados perinatais e tornou-se um produto de consumo, de tal maneira que as taxas são menores entre as mulheres com menor poder aquisitivo e aumentam à medida que o recurso financeiro é maior. ${ }^{15}$

Dentre os fatores associados à realização do parto vaginal e cirúrgico, evidencia-se que a chance de realização da cesárea triplica entre as mulheres que desejam esse procedimento; já no serviço privado as chances são 18 vezes mais elevadas para sua realização. Entre as multíparas, tanto para mulheres com seguimento pré-natal no SUS quanto no serviço privado, o histórico de parto operatório anterior aumenta em 11 vezes a chance de ter uma nova cesariana. ${ }^{16}$

Nesta mesma pesquisa, identificou-se que a mulher atendida na saúde suplementar possui maior poder de decisão, quanto à possibilidade de escolha de profissional e equipe que prestará a assistência, bem como do local e data de nascimento do bebê e por meio de cesárea eletiva. A conveniência em programar o nascimento do filho e evitar a dor do parto foram os motivos mencionados como justificativa para a realização da cesárea agendada. ${ }^{16}$

Fato também observado em um estudo no município do Vale do Taquari, São Paulo, onde verificou que mulheres com parto cesariana anterior e relatou que o motivo da opção por esta via de parto foi a indicação médica. As mulheres que escolheram o parto vaginal foram influenciadas pelos familiares (43\%), no entanto, quem mais contribuiu para a escolha pelo parto 
Orientações sobre parto no pré-natal de alto risco nos serviços de saúde | 14

cesariana foi o médico (31\%). Observou-se, também, que as mulheres não estavam preparadas para o parto vaginal, pois não receberam informações que diminuíssem os seus medos, e, também, não foram informadas sobre as complicações inerentes ao parto cesárea para a sua saúde e do bebê. ${ }^{17}$

Os resultados encontrados neste estudo não diferem dos achados em outras pesquisas ${ }^{15-}$ ${ }^{16}$, sendo que a mulher, muitas vezes, é convencida para o parto cesariana como a melhor opção. O diagnóstico de alto risco não pode ser decisivo para o desfecho do parto cesariana. Nesta sequência, o profissional que conduz o pré-natal deve favorecer abertura para o esclarecimento das dúvidas e anseios da mulher sobre a via de parto em qualquer momento do pré-natal.

Observou-se que o adiamento das explicações sobre o parto esteve presente durante as consultas de pré-natal, fato este que pode intensificar a dúvida das mulheres, em que as mesmas não têm o atendimento às suas incertezas no momento da sua necessidade, o que pode fazer com que a mulher recorra a outros meios de informações.

No presente estudo, as orientações sobre o parto cesariana foram vistas como uma estratégia de prevenção de agravos fetais. Entretanto, pode-se inferir que, muitas vezes, essa via é prescrita mesmo com condições de saúde estável da mulher e do feto. Torna-se importante o resgate quanto a indicações para o parto cesariana e as possibilidades para o parto vaginal em gestações de alto risco, para que seja uma estratégia efetiva para prevenção de agravos e não utilizada rotineiramente.

As contraindicações para indução do parto em gravidez de alto risco e, consequentemente, indicação de parto cesariana são: O sofrimento fetal agudo, a cicatriz segmentar de repetição (duas ou mais), as situações de urgência, as apresentações fetais anômalas, a desproporção céfalo-pélvica absoluta, a placenta prévia, a presença de incisão uterina corporal prévia, o carcinoma invasivo do colo, o prolapso de cordão, a herpes genital ativa e a obstrução do canal de parto. ${ }^{1}$ 
A cesariana, quando bem indicada, reduz a morbimortalidade materna e perinatal, porém deve-se ressaltar que seus riscos são maiores do que o parto vaginal. A sua prática indiscriminada, além do consumo de recursos do sistema de saúde, viola o exercício dos direitos sexuais e reprodutivos das mulheres. ${ }^{18}$

O não estímulo ao parto vaginal é fortemente observado no pré-natal, oferecido pelo serviço privado, pelo acompanhamento ser realizado exclusivamente pelo médico. Enquanto isso, no SUS, em muitos municípios, as consultas são alternadas entre médico e enfermeiro. Torna-se importante o rompimento do paradigma social sobre a cesariana. ${ }^{16}$

Um estudo de coorte, que avaliou as repercussões entre risco gestacional e tipo de parto, concluiu que a cesariana não influenciou diretamente o resultado materno, mas aumentou as chances de um resultado neonatal desfavorável. ${ }^{19}$

Ressalta-se a escassez de material encontrado sobre associação da gestação de risco e via de parto. Pode-se inferir que a insuficiência de evidência científica sobre a temática pode prejudicar as ações dos profissionais durante o seguimento e a assistência pré-natal. As informações sobre via de parto durante a gestação de alto risco deve ser uma prática considerável para melhoria da assistência materna e neonatal. A gestação é um momento favorável para quebra de paradigmas, por meio do estabelecimento do vínculo entre profissional, gestante e familiares.

O presente estudo desvelou que quando a gestante não se sente orientada o suficiente sobre suas dúvidas, essa recorre a outras fontes de informações, como o uso da internet para sanar suas dúvidas. Essa é uma ferramenta utilizada pelas gestantes no esclarecimento de dúvidas, quanto ao conteúdo buscado, não se direcionando para a própria saúde da mulher, mas à saúde e bem-estar fetal. ${ }^{20}$

As participantes do estudo buscaram as informações sobre as vias de parto, para explicar a realidade que as cercaram durante o período gestacional no alcance de se criar as 
Orientações sobre parto no pré-natal de alto risco nos serviços de saúde | 16

representações sociais. A interpretação da realidade proporciona o conhecimento social elaborado, entretanto, para que esse processo aconteça durante a gestação, é preciso criar espaços e explorar sua elaboração. Os profissionais que realizam assistência pré-natal de alto risco, quando não orientam sobre o fenômeno do parto, geram impossibilidades ou dificuldades para a elaboração do conhecimento da vida cotidiana.

O fenômeno de gestar pode fazer com que a mulher não se perceba como um ser que também precisa de cuidados, tanto quanto o bebê, sendo que os exames de imagem são reconhecidos como substitutos das orientações profissionais. O profissional que acompanha o pré-natal de alto risco deve inserir a gestante para o cuidado próprio, favorecendo o entendimento da mulher sobre a importância de sua própria saúde.

Assim, o estímulo para o empoderamento da gestante de alto risco, por meio dos profissionais pré-natalistas, é importante para que a mulher se perceba como uma pessoa essencial no processo de gestar. A mulher deve receber orientações quanto ao autocuidado, à promoção e à prevenção de agravos, para o próprio bem-estar, assim o serviço de saúde pode incentivar e orientar quanto ao cuidado adequado durante a sua gestação.

O empoderamento feminino é iniciado a partir de ações educativas, tendo como objetivo o desenvolvimento de atitudes, habilidades e autoconhecimento, para que a mulher possa assumir decisões e responsabilidades com sua saúde. Durante o pré-natal, trabalho de parto, parto e nascimento, é possível que a gestante estabeleça um poder de decisão sobre os momentos da sua parturição. Essas dimensões estão relacionadas à escolha do local onde o bebê irá nascer, do acompanhante, do profissional que deverá assisti-la, das tecnologias não invasivas de cuidado aplicadas ao processo de gestar, parir e nascer. ${ }^{21}$

Nesse sentido, o plano de parto é um recurso que deve ser inserido para o encorajamento das gestantes, o qual ainda é desconhecido por profissionais de saúde, hospitais e maternidades, fazendo com que não aconteça seu uso. ${ }^{22}$ Ressalta-se que quando a gestante é atendida quanto às 
suas necessidades individuais, criam-se espaços para o conhecimento organizado, a partir dos acontecimentos da vida cotidiana que ocorrem de acordo com a exploração do processo psicossocial e refletem no posicionamento da gestante quanto ao próprio cuidado e da facilidade para a comunicação com o profissional.

A visita à maternidade é uma ação que ainda está em processo de implementação, porém, se bem implantada, pode trazer maior segurança e conforto à mulher, pela oportunidade de conhecer o local que seu filho irá nascer. Conforme a Lei ${ }^{\circ} 11.634$, de 27 de dezembro de 2007, toda gestante assistida pelo SUS tem direito ao conhecimento e vinculação prévia da maternidade na qual será realizado seu parto e onde será atendida em casos de intercorrências na gestação. ${ }^{23}$

Salienta-se que orientações recebidas durante o pré-natal para o momento do parto, devem ter o direcionamento para o esclarecimento de dúvidas, além de informações pertinentes ao ciclo gravídico-puerperal. Por meio da Teoria das Representações Sociais, identificam-se as necessidades das informações sobre o parto na assistência pré-natal de alto risco e, a possibilidade de enaltecer o processo cognitivo da gestante, almejando o conhecimento elaborado.

O vínculo formado entre profissional e gestante fornece subsídios para que a gestação seja um momento de construção de um processo educativo. Assim, os conhecimentos sobre sua saúde serão construídos a cada consulta, favorecendo desfechos materno-fetais mais positivos e melhoria da qualidade no cuidado.

Dentre as limitações do presente estudo, ressaltam-se sua realização em apenas uma das maternidades da cidade e com um grupo de mulheres no período da pesquisa, o que restringe a análise da assistência pré-natal oferecida de modo geral, considerando que há realidades bastante diferentes nas unidades de saúde, ambulatórios de alto risco e consultórios médicos. 
Entretanto, os resultados revelaram aspectos importantes para assistência gestacional de alto risco.

\section{Conclusão}

Ao analisar a percepção de puérperas que foram acompanhadas como gestantes de alto risco na saúde pública e privada à luz da Teoria das Representações Sociais, revelou-se que as orientações recebidas foram direcionadas à saúde e bem-estar fetal, identificação de situações de risco ao bebê, evidenciadas nas urgências que poderiam resultar em um parto cesáreo prematuro e complicações ao concepto. Houve similaridade das orientações recebidas nos dois tipos de serviços de saúde, assim como uma lacuna na reelaboração da forma de conhecimento da gestante sobre o momento do parto. As orientações essenciais para o parto não foram supridas no grupo social de gestantes de alto risco, resultando na necessidade de busca do aprendizado quanto aos acontecimentos da vida cotidiana.

Assim, reforça-se a necessidade, em ambos os serviços de saúde, de aprimoramento das ações educativas, visando a melhoria da qualidade da assistência pré-natal para as gestantes de alto risco, com implantação de protocolos específicos que abranjam a assistência pré-natal na sua totalidade, buscando preparar a mulher para o momento da internação, para o trabalho de parto e o próprio parto, independentemente do local onde for realizado.

Considera-se que incluir as representações sociais das puérperas na avaliação das orientações recebidas, durante a consulta no pré-natal de alto risco, será uma forma de adotar medidas que assegurem a qualidade da assistência no ciclo gravídico puerperal. Sugere-se a realização de novos estudos sobre orientações no pré-natal de alto risco, com diferentes desenhos metodológicos, buscando uma melhor compreensão, favorecendo melhorias para a assistência pré-natal às gestantes com diagnóstico de alto risco. 


\section{Referências}

1. Ministério da Saúde (BR), Secretaria de Atenção à Saúde, Departamento de Ações Programáticas Estratégicas. Gestação de alto risco: manual técnico [Internet]. 5ª ed. Brasília (DF): Ministério da Saúde; 2010 [acesso jul 2019 10]. Disponível em: http://bvsms.saude.gov.br/bvs/publicacoes/gestacao_alto_risco.pdf

2. Lansky S, Friche AAL, Silva AAM, Campos D, Bittencourt SDA, Carvalho ML, et al. Pesquisa Nascer no Brasil: perfil da mortalidade neonatal e avaliação da assistência à gestante e ao recém-nascido. Cad Saúde Pública. 2014;30(1):192-207. doi: 10.1590/0102-311X00133213

3. Nunes JT, Gomes KRO, Rodrigues MTP, Mascarenhas MDM. Qualidade da assistência pré-natal no Brasil: revisão de artigos publicados de 2005 a 2015. Cad Saúde Colet. 2016;24(2):252-61. doi: 10.1590/1414$462 X 201600020171$

4. Polgliane RBS, Leal MC, Amorim MHC, Zandonade E, Santos Neto ET. Adequação do processo de assistência pré-natal segundo critérios do Programa de Humanização do Pré-natal e Nascimento e da Organização Mundial de Saúde. Ciênc Saúde Colet. 2014;19(7):1999-2010. doi: 10.1590/141381232014197.08622013

5. Tomasi E, Fernandes PAA, Fischer T, Siqueira FCV, Silveira DS, Thumé E, et al. Qualidade da atenção pré-natal na rede básica de saúde do Brasil: indicadores e desigualdades sociais. Cad Saúde Pública. 2017;33(3):e00195815. doi: 10.1590/0102-311x00195815

6. Guimarães TA, Rocha AJSC, Rodrigues WB, Pasklan ANP. Mortalidade materna no Brasil entre 2009 e 2013. Rev Pesq Saúde [Internet]. 2017 [acesso 2019 out 10];18(2):81-5. Disponível em: http://www.periodicoseletronicos.ufma.br/index.php/revistahuufma/article/view/8381

7. Silva ALA, Mendes ACG, Miranda GMD, Souza WV. A qualidade do atendimento ao parto na rede pública hospitalar em uma capital brasileira: a satisfação das gestantes. Cad Saúde Pública. 2017;33(12):e00175116. doi: 10.1590/0102-311x00175116

8. Domingues RMSM, Viellas EF, Dias MAB, Torres JA, Theme-Filha MM, Gama SGN, et al. Adequação da assistência pré-natal segundo as características maternas no Brasil. Rev Panam Salud Publica [Internet]. 2015 [acesso 2019 set 15];37(3):140-7. Disponível em: https://scielosp.org/article/rpsp/2015.v37n3/140-147/

9. Jodelet D. Representações sociais: um domínio em expansão. Rio de Janeiro: Guanabara Koogan; 2002.

10. Vala J, Castro P. Pensamento social e representações sociais. In: Vala J, Monteiro MB. Psicologia Social. 9ª ed. Lisboa: Fundação Calouste Gulbenkian; 2013. p. 569-602.

11. Secretaria de Estado da Saúde do Paraná, Superintendência de Atenção à Saúde. Linha Guia Rede Mãe Paranaense [Internet]. Curitiba: SESA; 2014 [acesso em 2019 jul 10]. Disponível em: 
Orientações sobre parto no pré-natal de alto risco nos serviços de saúde | 20

http://www.saude.pr.gov.br/sites/default/arquivos_restritos/files/documento/2020-

04/linhaguiamaeparanaense_final_2017.pdf

12. Fontanella BJB, Ricas J, Turato ER. Amostragem por saturação em pesquisas qualitativas em saúde: contribuições teóricas. Cad Saúde Pública. 2008;24(1):17-27. doi: 10.1590/S0102-311X2008000100003

13. Bardin L. Análise de conteúdo. São Paulo: Edições 70; 2011.

14. Viellas EF, Domingues RMSM, Dias MAB, Gama SGN, Theme-Filha MM, Costa JV, et al. Assistência pré-natal no Brasil. Cad Saúde Pública. 2014;30(1):85-100. doi: 10.1590/0102-311X00126013

15. Paris GF, Monteschio LVC, Oliveira RR, Latorre MRDO, Pelloso SM, Mathias TAF. Tendência temporal da via de parto de acordo com a fonte de financiamento. Rev Bras Ginecol Obstet. 2014;36(12):548-54. doi: 10.1590/So100-720320140005038

16. Oliveira RR, Melo EC, Novaes ES, Ferracioli PLRV, Mathias TAF. Factors associated to caesarean delivery in public and private health care systems. Rev Esc Enferm USP. 2016;50(5):733-40. doi: 10.1590/s0080-623420160000600004

17. Weidle WG, Medeiros CRG, Grave MTQ, Dal Bosco SM. Escolha da via de parto pela mulher: autonomia ou indução? Cad Saúde Colet. 2014;22(1):46-53. doi: 10.1590/1414-462X201400010008

18. Cabral FB, Hirt LM, Van der Sand ICP. Atendimento pré-natal na ótica de puérperas: da medicalização à fragmentação do cuidado. Rev Esc Enferm USP. 2013;47(2):281-7. doi: 10.1590/S008062342013000200002

19. Reis ZSN, Lage EM, Eura M, Aguiar RALP, Gaspar JS, Vitral GLN, et al. Associação entre risco gestacional e tipo de parto com as repercussões maternas e neonatais. Rev Bras Ginecol Obstet [Internet]. 2014 [acesso em 2019 out 13];36(2):65-71. Disponível em: http://www.scielo.br/pdf/rbgo/v36n2/0100-7203-rbgo-36-02-00065.pdf

20. Calderon TM, Cestari MEW, Dobkowski AC, Cavalheiro MD. O uso da Internet como ferramenta de apoio ao esclarecimento de dúvidas durante a gestação. J Health Biol Sci (Online). 2016;3(2):18-22. doi: 10.12662/2317-3076jhbs.v4i1.523.p18-22.2016

21. Suárez-Cortés M, Armero-Barranco D, Canteras-Jordana M, Martínez-Roche ME. Uso e influencia de los Planes de Parto y Nacimiento en el proceso de parto humanizado. Rev Latinoam Enferm (Online). 2015;23(3):520-6. doi: 10.1590/0104-1169.0067.2583

22. Mouta RJO, Silva TMA, Melo PTS, Lopes NS, Moreira VA. Plano de parto como estratégia de empoderamento feminino. Rev Baiana Enferm. 2017;31(4):e20275. doi: 10.18471/rbe.v31i4.20275

23. BRASIL. Ministério da Saúde. Lei no 11.634, de 27 de dezembro de 2007. Dispõe sobre o direito da gestante ao conhecimento e vinculação à maternidade onde receberá assistência no âmbito Sistema 
Único de Saúde. Brasília, DF: Ministério da Saúde, 2007. Disponível em: http://www.planalto.gov.br/ccivil_03/_Ato2007-2010/2007/Lei/L11634.htm. Acesso em: 10 jul. 2019.

Editor Científico Chefe: Cristiane Cardoso de Paula

Editor Científico: Tânia Solange Bosi de Souza Magnago

\section{Autor correspondente}

Fabiana Fontana Medeiros

E-mail: fontana.fabi@hotmail.com

Endereço: Av. Robert Kock, 60. Operária. Londrina - PR

CEP: 86038-350

\section{Contribuições de Autoria}

1 - Juliana Carvalho Lourenço

Concepção e planejamento do projeto de pesquisa, obtenção, análise e interpretação dos dados, redação e revisão crítica.

\section{2 - Fabiana Fontana Medeiros}

Análise e interpretação dos dados, redação e revisão crítica.

\section{3 - Mariana Haddad Rodrigues}

Análise e interpretação dos dados, redação e revisão crítica.

\section{4 - Rosângela Aparecida Pimenta Ferrari}

Redação e revisão crítica.

5 - Deise Serafim

Redação e revisão crítica.

\section{6 - Alexandrina Aparecida Maciel Cardelli}

Concepção e planejamento do projeto de pesquisa, análise e interpretação dos dados, redação e revisão crítica.

\section{Como citar este artigo}

Lourenço JC, Medeiros FF, Rodrigues MH, Ferrari RAP, Serafim D, Cardelli AAM. Orientações sobre parto no prénatal de alto risco nos serviços de saúde Rev. Enferm. UFSM. 2020 [Acesso em: Anos Mês Dia]; vol.10 e85: 1-21. DOI:https://doi.org/10.5902/2179769241357 